\title{
Homogenization scheme for acoustic metamaterials
}

\author{
Min Yang, ${ }^{1, *}$ Guancong Ma, ${ }^{1}$ Ying Wu, ${ }^{3}$ Zhiyu Yang, ${ }^{1}$ and Ping Sheng ${ }^{1,2, \dagger}$ \\ ${ }^{1}$ Department of Physics, Hong Kong University of Science and Technology, Clear Water Bay, Kowloon, Hong Kong, China \\ ${ }^{2}$ Institute of Advanced Study, Hong Kong University of Science and Technology, Clear Water Bay, Kowloon, Hong Kong, China \\ ${ }^{3}$ Division of Computer, Electrical and Mathematical Science and Engineering, \\ King Abdullah University of Science and Technology, Thuwal, Saudi Arabia
}

(Received 27 December 2013; revised manuscript received 11 February 2014; published 26 February 2014)

\begin{abstract}
We present a homogenization scheme for acoustic metamaterials that is based on reproducing the lowest orders of scattering amplitudes from a finite volume of metamaterials. This approach is noted to differ significantly from that of coherent potential approximation, which is based on adjusting the effective-medium parameters to minimize scatterings in the long-wavelength limit. With the aid of metamaterials' eigenstates, the effective parameters, such as mass density and elastic modulus can be obtained by matching the surface responses of a metamaterial's structural unit cell with a piece of homogenized material. From the Green's theorem applied to the exterior domain problem, matching the surface responses is noted to be the same as reproducing the scattering amplitudes. We verify our scheme by applying it to three different examples: a layered lattice, a two-dimensional hexagonal lattice, and a decorated-membrane system. It is shown that the predicted characteristics and wave fields agree almost exactly with numerical simulations and experiments and the scheme's validity is constrained by the number of dominant surface multipoles instead of the usual long-wavelength assumption. In particular, the validity extends to the full band in one dimension and to regimes near the boundaries of the Brillouin zone in two dimensions.
\end{abstract}

DOI: 10.1103/PhysRevB.89.064309

PACS number(s): 43.20.+g, 43.40.+s, 46.40.-f, 62.30.+d

\section{INTRODUCTION}

The emergence of metamaterials has been based on the premise that the collective behavior of local resonances can lead to effective material parameter values not found in nature. By controlling the microstructural heterogeneities, exotic dynamic responses at the macro- or mesoscale can be achieved, and their characteristics have significantly broadened the horizon for elastic/acoustic wave manipulations. Novel phenomena, such as focusing and subwavelength imaging [1-4], near-field amplification [5], cloaking [6-9], localization of ultrasound [10], one-way transmission [11-13], as well as superabsorption [14] were proposed or were experimentally demonstrated. At the core of these phenomena are the resonance-induced and frequency-dependent effective material characteristics, such as highly anisotropic constitutive parameters [1,15], zero refractive index [16-19], negative mass [20-23], negative bulk modulus [24,25] or shear modulus [26], as well as simultaneously negative mass and modulus [18,27-30].

However, accompanying metamaterials' exotic effective parameter values is a challenge to the classical homogenization theory, which is based on the philosophy of adjusting the effective parameter values so as to minimize scatterings at the long-wavelength limit. Such a basic viewpoint is inconsistent with the fact that the novel effective parameter values of metamaterials arise mainly from the resonant scatterings. In particular, the novel properties of the metamaterials are usually at frequencies beyond the low frequency, or the long-wavelength limit, that is the validity constraint of the conventional homogenization approaches [31]. There are efforts to extend the conventional homogenization to higher frequencies

\footnotetext{
*Corresponding author: erwinstu@ust.hk

†Corresponding author: sheng@ust.hk
}

by a scheme denoted as "two-scale asymptotic" [32,33] in which a separate set of expansions (different from the longwavelength asymptotic) is applied near the standing-wave modes at the edges of the Brillouin zones (BZs) for periodic structures. The effective-medium characteristics in the pass bands can thereby be approached from two directions. There are also some nonasymptotic homogenization approaches for metamaterials. For Bloch-type electromagnetic (EM) waves in periodic media, a field-averaging homogenization scheme was proposed [34]. A broadband homogenization scheme was introduced [35] that considers the homogenization problem as averaging over the responses resulting from suitable source distributions. For elastic/acoustic systems, a general method for either periodic or random structures was presented [36], based on ensemble averaging of the response functions in which the system is driven by a specified body force so that the effective parameters are functions of both wave number and frequency. Enhanced versions of classical coherent potential approximation (CPA) have also been applied to both electromagnetic and elastic/acoustic metamaterials [37,38]; equivalent results were also obtained from matching the scattered waves by homogeneous scatterers with the predictions of the multiple-scattering formalism [39-41]. These schemes are noted to operate under the assumption of the wavelength being much larger than the relevant feature size. We should be reminded that, in the traditional homogenization schemes, the long-wavelength limit implicitly implies minimal scatterings since, for classical waves, the Rayleigh scattering cross section approaches zero in that limit. Hence, the long-wavelength limit is intrinsically consistent with the basic premise of minimizing scatterings in the traditional homogenization scheme. However, once there are local resonators, such as the case in metamaterials, the resonant scatterings differ significantly from Rayleigh scatterings. In particular, the fact that the wavelength is large compared to the feature size of each resonator no longer guarantees weak scattering. 
In this paper, as a generalization of the specialized technique reported in our previous paper [29], we propose an approach to homogenize acoustic metamaterials based on matching the lowest-order scattering amplitudes arising from a finite volume (or a structural unit) of the relevant metamaterial with that of a homogenized material whose material parameters are the desired quantities to be determined. From the Green's theorem applied to the exterior domain problem, matching the scattering amplitudes is the same as matching the interfacial responses of the relevant finite volume. The surface responses of a heterogeneous structural unit, in turn, may be formally calculated based on its eigenstates, which can be obtained numerically, e.g., such as by the finite element method (FEM). The problem can thereby be converted into a set of response equations for the unknown material parameters. We show that the relevant effective parameters can be explicitly solved as functions of frequency, and the homogenized effective medium provides an exact match to the motions at the boundaries of structural units (SUs) and, hence, the scattering amplitudes with that of the actual metamaterial. The advantage of this approach is that it is conceptually clear cut and focuses on the resonant scatterings that are the source of metamaterials' exotic behaviors. It can ensure validity over a broad range of frequencies: In one dimension (1D), the theory is valid for all frequencies, and in two dimensions (2D), the validity of the theory is shown to be almost over the entire Brillouin zone, even when the effective wavelength is comparable to the size of the unit cell at the Brillouin-zone boundary. Such accuracy is achieved by matching only the lowest two orders of scattering amplitudes (surface motions).

We demonstrate the effectiveness of the proposed approach through three examples. The first is a two-phase layered lattice in which one phase is stiff and heavy while the other phase is an elastic medium. The wave propagation characteristics and the relevant wave fields, calculated from the homogenized effective parameters, are shown to yield excellent agreement with numerical simulations. The second example is a $2 \mathrm{D}$ hexagonal lattice of concentric cylinders. The homogenized medium predictions are compared with numerical simulations from FEM and multiple-scattering theory (MST) with excellent agreement. The third example is the membrane-type metamaterial with a decorated-membrane resonator as the unit cell of a planar array. Again the comparison of the transmission/reflection characteristics with the experimental results from impedance tube measurements shows excellent agreement.

In what follows, derivation of the homogenization scheme is presented in Sec. II with the application to 1D scalar waves discussed in Sec. II B and the application to the 2D case discussed in Sec. IIC. Examples and results are given in Sec. III. In Sec. III A, we show and discuss the 1D layered lattice, followed by the 2D hexagonal lattice of concentric cylinders presented in Sec. III B. The case for the decoratedmembrane resonator is shown in Sec. IIIC. We conclude in Sec. IV with a summary of results and some comments on the prospect of generalizations.

\section{METAMATERIAL HOMOGENIZATION}

Metamaterials are usually composed of a finite number of similar units comprising inner heterogeneities, shown (a)

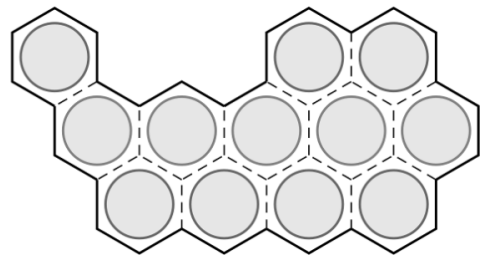

(b)

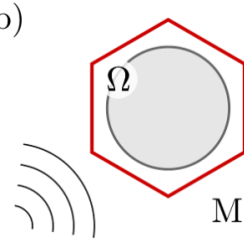

FIG. 1. (Color online) (a) Schematic of the unit cells and their boundaries for a typical metamaterial. The inner heterogeneous scatterers are denoted by gray disks, the external boundaries are marked by black solid lines, and the internal unit boundaries are delineated by dashed lines. (b) The relevant SU (marked $\Omega$ ) of the metamaterial. Its boundary is denoted by the red lines. The incident waves are illustrated as circular wave fronts in the medium $M$, external to the SU.

schematically in Fig. 1(a). The relevant wave propagation characteristics as well as the consequent effective wave vector $\bar{k}$ [42] and impedance $\bar{Z}$ at the interface (delineated by the black solid line) are completely determined by the scatterings of its single SU [as delineated in Fig. 1(b) by the red line]. As shown in what follows, the most relevant properties of the SU to our homogenization scheme are the surface responses characterized by its Green's function with both arguments evaluated on the boundary- $G\left(\xi^{\prime}, \xi\right)$, where $\xi$ and $\xi^{\prime}$ denote coordinates on the SU's boundary $\partial \Omega$ [the red line in Fig. 1(b)]. Below we show that the surface responses are directly related to the scatterings by the SU.

Consider a SU [region $\Omega$ in Fig. 1(b)] embedded in a medium $M$ containing a certain background incident wave (displacement field) $\Phi_{\text {in }}$. Since, in this paper, we consider only scalar acoustic waves, the displacement involved will comprise only one (vector) component. The medium $M$ can be either the neighboring units (for the internal units) or the external environmental medium of the metamaterial (for the surface units). By applying the Green's theorem to the medium [43], the scattering field $\Phi_{\mathrm{sc}}$ from the $\mathrm{SU}$ is related to the interfacial normal stress $\psi_{n}(\xi)$ on the surface $\partial \Omega$ as

$$
\Phi_{\mathrm{sc}}\left(x^{\prime}\right)=\oint_{\partial \Omega} G_{\mathrm{M}}\left(x^{\prime}, \xi\right) \psi_{n}(\xi) d \xi,
$$

in which $x^{\prime}$ represents the bulk coordinate in the medium external to the SU and its Green's function $G_{\mathrm{M}}$ satisfies the homogeneous Neumann boundary condition. One can similarly apply the Green's theorem on the SU to relate the normal stress $\psi_{n}$ with the total displacement $\Phi_{\text {in }}+\Phi_{\text {sc }}$ on $\partial \Omega$,

$$
\psi_{n}(\xi)=-\oint_{\partial \Omega} G^{-1}\left(\xi, \xi^{\prime}\right)\left[\Phi_{\mathrm{in}}\left(\xi^{\prime}\right)+\Phi_{\mathrm{sc}}\left(\xi^{\prime}\right)\right] d \xi^{\prime},
$$

where the Green's function $G$ is internal to the SU. It also obeys the homogeneous Neumann boundary condition, and its inverse $G^{-1}$ satisfies $\int_{\Omega} G^{-1}\left(x, x^{\prime}\right) G\left(x^{\prime}, x^{\prime \prime}\right) d x^{\prime}=\delta\left(x, x^{\prime \prime}\right)$. The minus sign in front of Eq. (1b) is from Newton's third law. By substituting Eq. (1b) into Eq. (1a), we obtain an expression for the scattering field as

$$
\begin{aligned}
\Phi_{\mathrm{sc}}(x)= & -\oiint_{\partial \Omega} G_{\mathrm{M}}(x, \xi) G^{-1}\left(\xi, \xi^{\prime}\right)\left[\Phi_{\mathrm{in}}\left(\xi^{\prime}\right)\right. \\
& \left.+\Phi_{\mathrm{sc}}\left(\xi^{\prime}\right)\right] d \xi d \xi^{\prime} .
\end{aligned}
$$


In Eq. (2), it is seen that the SU contributes only in terms of its surface responses $G\left(\xi, \xi^{\prime}\right)$. By employing a set of orthonormal surface modes $\left\{\varphi_{\alpha}\right\}$ defined on the SU's surface $\partial \Omega$, these surface responses can be expressed in terms of scalar numbers,

$$
G_{\alpha \beta} \equiv \oiint_{\partial \Omega} \varphi_{\alpha}^{*}\left(\xi^{\prime}\right) G\left(\xi^{\prime}, \xi\right) \varphi_{\beta}(\xi) d \xi^{\prime} d \xi,
$$

where the integrals are over the entire surface.

The wave characteristics of metamaterial's SU, with all its structural complexities, may be calculated numerically in terms of its eigenvalues and eigenfunctions. The Green's function can then be obtained in the form of eigenfunction expansion, and the $G_{\alpha \beta}$ can be explicitly evaluated (Sec. II A). Effective material parameter values can then be obtained at each frequency by solving the following equation:

$$
G_{\alpha \beta}=\bar{G}_{\alpha \beta}(\bar{k}, \bar{Z}),
$$

with $\bar{G}$ denoting the Green's function of the homogenized material characterized by the effective parameters whose values are to be determined. Notice that, as the unknown variables, $\bar{k}$ and $\bar{Z}$ are usually not just two simple scalars but can have a large number of components when complexities (such as anisotropy and polarizations) are present in the effective constitutional relations-Newton's second law and Hooke's law [44]. However, the number of equations as expressed by Eq. (4) must be consistent with the square of the number of surface modes, thus, generally infinite. It follows that, to guarantee the solution of Eq. (4), there must be truncation in the number of surface modes to be considered so that it matches the number of effective material parameters. Since we would like to first account for the lower-frequency behaviors, therefore, only the lowest multipoles will be considered. In this manner, there is a natural upper frequency limit beyond which our scheme becomes inaccurate.

It should be noted that, although the proposed scheme is for systems with resonant inclusions, it can be equally effective for nonresonant systems. The only difference between the two cases lies in the fact that, for metamaterials, the eigenfunctions of the system usually already have the monopole or dipole symmetry, hence, only a few eigenfunctions are needed in the Green's function expansion, shown below. For the nonresonant systems, more eigenfunctions may be needed in order to attain the desired accuracy.

\section{A. Expansion of the Green's function by resonant eigenfunctions}

Metamaterial owes its novel features to the collective behaviors of arrays of local resonators. A suitable mathematical description of such behaviors would be the Green's function expansion based on the eigenfunctions of its SU. Let $\left\{\phi_{i}\right\}$ be the set of normalized eigenstates of the SU under the homogeneous Neumann boundary condition, i.e., under the force-free condition. The Green's function has an expansion form as $[29,45]$

$$
G\left(x^{\prime}, x\right)=\sum_{i} \frac{\phi_{i}\left(x^{\prime}\right) \phi_{i}^{*}(x)}{\rho_{i}\left(\omega_{i}^{2}-\omega^{2}\right)},
$$

where $\omega$ denotes angular frequency. For each eigenstate $\phi_{i}(x)$, an average mass density is defined as

$$
\rho_{i} \equiv \int_{\Omega} \phi_{i}^{*}(x) \rho(x) \phi_{i}(x) d x,
$$

with $\rho(x)$ being the local mass density. Here, $\omega_{i}$ is the corresponding eigenfrequency. The summation is over all the eigenstates.

It should be noted that, although the boundary condition for $\phi_{i}$ is assumed to be force free, the solution to those problems where the boundary forces are nonzero can also be obtained from the above Green's function through integration with the inhomogeneous term.

In accordance with its definition Eq. (3), the surface responses of the SU can be expressed as

$$
G_{\alpha \beta}=\sum_{i} \frac{\oint_{\partial \Omega} \varphi_{\alpha}^{*}\left(\xi^{\prime}\right) \phi_{i}\left(\xi^{\prime}\right) d \xi^{\prime} \oint_{\partial \Omega} \phi_{i}^{*}(\xi) \varphi_{\beta}(\xi) d \xi}{\rho_{i}\left(\omega_{i}^{2}-\omega^{2}\right)}
$$

From Eqs. (5) and (7), it is seen that each term in the summation is only dominant in the frequency domain around its eigenfrequency $\omega_{i}$. Response of the SU is strongest at these resonance frequencies, accompanied with a jump of $\pi$ in phase. Hence, instead of the infinite summations, for the purpose of investigating a finite frequency range, only several eigenstates in the relevant range are needed. The inclusion of additional eigenmodes, beyond those in the relevant frequency range, can improve the accuracy. We would like to note that, between two adjacent eigenmodes, there is always an antiresonance at which the SU is decoupled to external waves and $G_{\alpha \beta}=0$.

\section{B. Homogenization of one-dimensional systems}

Explicit relations between $\bar{G}_{\alpha \beta}$ and its effective parameters $(\bar{k}, \bar{Z})$ on the right-hand side of Eq. (4) are given in this section for $1 \mathrm{D}$ systems.

For scalar waves, a 1D SU with a length of $2 a$ has only two independent surface modes,

$$
\begin{aligned}
& \varphi_{0}=[\delta(x-a)+\delta(x+a)] / \sqrt{2}, \\
& \varphi_{1}=[\delta(x-a)-\delta(x+a)] / \sqrt{2},
\end{aligned}
$$

where $\varphi_{0}\left(\varphi_{1}\right)$ is related to the monopolar (dipolar) scattering and $\delta$ is the Dirac $\delta$ function. In the parity symmetric cases, except for the two trivial ones $G_{01}=G_{10}=0$, the SU's surface responses are completely described by the following two values as defined by Eq. (7):

$$
\begin{aligned}
G_{00} & =\sum_{i} \frac{\phi_{i}^{*}(a)\left[\phi_{i}(a)+\phi_{i}(-a)\right]}{\rho_{i}\left(\omega_{i}^{2}-\omega^{2}\right)}, \\
G_{11} & =\sum_{i} \frac{\phi_{i}^{*}(a)\left[\phi_{i}(a)-\phi_{i}(-a)\right]}{\rho_{i}\left(\omega_{i}^{2}-\omega^{2}\right)} .
\end{aligned}
$$

Hence, Eq. (4) implies only two equations.

For the homogenized material, the governing equation is given by

$$
\left(\frac{\partial^{2}}{\partial x^{2}}+\bar{k}^{2}\right) \bar{\phi}(x)=0
$$


with the accompanying boundary condition,

$$
\vec{\psi}(\xi)=\omega \bar{Z} \partial \bar{\phi}(x) /\left.\partial(\bar{k} x) \cdot \hat{n}\right|_{x=\xi}
$$

for external surface traction $\vec{\psi}$, where $\hat{n}$ denotes the outward normal unit vector. The two effective parameters are given by $\bar{k}=\omega \sqrt{\bar{\rho} / \bar{C}}$ and $\bar{Z}=\sqrt{\bar{\rho} \bar{C}}$, where $\bar{\rho}$ denotes the effectivemass density and $\bar{C}$ denotes the effective modulus. There is equality between the number of equations and the number of unknown variables $\bar{\rho}$ and $\bar{C}$ in Eq. (4). This ensures that the $1 \mathrm{D}$ scalar wave system can be homogenized to all frequencies.

From the solution to Eq. (10), one can explicitly obtain the following expressions (details can be found in Appendix A):

$$
\bar{G}_{00}=-\frac{\cot (\bar{k} a)}{\omega \bar{Z}}, \quad \bar{G}_{11}=\frac{\tan (\bar{k} a)}{\omega \bar{Z}} .
$$

By substituting Eq. (11) into the homogenization condition Eq. (4), the effective wave vector $\bar{k}$ can be obtained as the solution of the following transcendental equation:

$$
G_{00} / G_{11}=-\cot ^{2}(\bar{k} a) .
$$

Consequently, the effective impedance $\bar{Z}$ is given by

$$
\bar{Z}=-\cot (\bar{k} a) /\left(\omega G_{00}\right) .
$$

Based on these two expressions, the two material parameters $\bar{\rho}$ and $\bar{Z}$ are determined by

$$
\bar{\rho}=\bar{Z} \bar{k} / \omega, \quad \bar{C}=\omega \bar{Z} / \bar{k} .
$$

It should be noted that the solution of Eq. (12a) can yield multiple solutions for $\bar{k}$. The natural choice is for $|\operatorname{Re}(\bar{k})|$ to be as small as possible. In a periodic system, this corresponds with the first Brillouin zone.

From Eq. (12a), it can be deduced that, even when the dissipation is absent in the system (both $G_{00}$ and $G_{11}$ are real), the effective wave vector $\bar{k}$ may still have an imaginary part, provided $G_{00} / G_{11}>0$. This indicates the existence of evanescent modes, i.e., band gaps. An extreme case is in the limit when $\bar{k} \rightarrow i \infty$ that corresponds to $G_{00}=G_{11}$. As demonstrated in Sec. IIIC, a small decay length can ensure the superattenuation phenomena in the membrane-type metamaterial in which sound waves with long wavelengths can be completely blocked by a thin layer of decorated membrane.

Direction of the phase velocity for the propagating waves relative to the direction of wave energy propagation, i.e., in the pass bands with $G_{00} / G_{11} \leqslant 0$, can be identified from Eq. (12a) by adding a small dissipation factor into the system. Small dissipation gives the effective wave vector a small positive imaginary part, i.e., $\bar{k}+i \varepsilon / a$ and, thereby, leads to an extra imaginary component on the right-hand side of Eq. (12a),

$$
2 i \varepsilon \cot (\bar{k} a) / \sin ^{2}(\bar{k} a) .
$$

The small dissipation also gives rise to a positive imaginary component for each of the two heterogeneous surface responses: $G_{00(11)}+i \beta g_{00(11)}$ [29] with $\beta$ being the infinitesimal dissipation coefficient and

$$
g_{00(11)} \equiv \sum_{i} \frac{\omega \phi_{i}^{*}(a)\left[\phi_{i}(a) \pm \phi_{i}(-a)\right]}{\rho_{i}\left(\omega_{i}^{2}-\omega^{2}\right)^{2}} .
$$

It follows from the above that an extra imaginary part would appear on the left-hand side of Eq. (12a) as

$$
-i \beta G_{00}\left(g_{11}-g_{00} G_{11} / G_{00}\right) / G_{11}^{2} .
$$

Since $G_{00} / G_{11} \leqslant 0$, the equality between the two imaginary parts Eqs. (13a) and (13b) means that $\bar{k}$ must be positive when $G_{00}<0$, i.e., the phase velocity is along the positive direction. Otherwise, $\bar{k}$ is negative when $G_{00}>0$. One can easily prove that, under this situation, both effective-mass density $\bar{\rho}$ and modulus $\bar{C}$ should be negative in their real parts (double negativity).

\section{Homogenization of isotropic two-dimensional systems}

In $2 \mathrm{D}$, the simplest isotropic SU is a circular disk. Its $\alpha$ th surface mode for scalar waves is

$$
\varphi_{\alpha}(\xi)=\delta(r-a) \cos (\alpha \theta) / \sqrt{a \pi},
$$

where $\delta$ denotes the Dirac $\delta$ function, $r$ is the radial coordinate, and $\xi=(a, \theta)$ denotes the surface coordinate, where $\theta$ is the angle measured from some fixed axis. For simplicity, we will consider only the lowest two surface modes - the monopolar case with $\alpha=0$ and the dipolar case with $\alpha=1$.

Under the above truncation, an isotropic SU has only two nontrivial scalars for its surface responses as defined by Eq. (7),

$$
\begin{aligned}
G_{00} & =\frac{a}{2 \pi} \sum_{i} \frac{\int_{0}^{2 \pi} \phi_{i}\left(a, \theta^{\prime}\right) d \theta^{\prime} \int_{0}^{2 \pi} \phi_{i}^{*}(a, \theta) d \theta}{\rho_{i}\left(\omega_{i}^{2}-\omega^{2}\right)}, \\
G_{11} & =\frac{a}{\pi} \sum_{i} \frac{\int_{0}^{2 \pi} \phi_{i}\left(a, \theta^{\prime}\right) \cos \theta^{\prime} d \theta^{\prime} \int_{0}^{2 \pi} \phi_{i}^{*}(a, \theta) \cos \theta d \theta}{\rho_{i}\left(\omega_{i}^{2}-\omega^{2}\right)} .
\end{aligned}
$$

With the above constraint, Eq. (4) is noted to consist of only two equations.

For homogenization, we consider an effective 2D isotropic scalar wave equation,

$$
\left(\frac{\partial^{2}}{\partial r^{2}}+\frac{1}{r} \frac{\partial}{\partial r}+\frac{1}{r^{2}} \frac{\partial^{2}}{\partial \theta^{2}}+\bar{k}^{2}\right) \bar{\phi}(r, \theta)=0,
$$

accompanied by the boundary condition for a surface traction $\vec{\psi}$ along the surface normal $\hat{n}$ pointing outward,

$$
\vec{\psi}(a, \theta)=\omega \bar{Z} \partial \bar{\phi}(r, \theta) /\left.\partial(\bar{k} r) \cdot \hat{n}\right|_{r=a} .
$$

The wave equation and its boundary condition are noted to contain two effective parameters $\bar{k}=\omega \sqrt{\bar{\rho} / \bar{C}}$ and $\bar{Z}=\sqrt{\bar{\rho} \bar{C}}$.

Analytical solutions for the two homogeneous surface responses of interest can be solved from Eq. (16) as (see Appendix B for details)

$$
\begin{aligned}
\bar{G}_{00} & =-J_{0}(\bar{k} a) /\left[\omega \bar{Z} J_{1}(\bar{k} a)\right], \\
\bar{G}_{11} & =\frac{\bar{k} a J_{1}(\bar{k} a)}{\omega \bar{Z}\left[\bar{k} a J_{0}(\bar{k} a)-J_{1}(\bar{k} a)\right]} .
\end{aligned}
$$

Here, $J_{0(1)}(\bar{k} a)$ is the Bessel function of the first kind with the order 0(1). Substitution of Eq. (17) into the homogenization condition Eq. (4) gives a transcendental equation for the 
effective wave vector $\bar{k}$ as

$$
\frac{G_{00}}{G_{11}}=\frac{J_{0}(\bar{k} a) J_{1}(\bar{k} a)-\bar{k} a J_{0}^{2}(\bar{k} a)}{\bar{k} a J_{1}^{2}(\bar{k} a)} .
$$

The effective impedance $\bar{Z}$ can be determined as

$$
\bar{Z}=-J_{0}(\bar{k} a) /\left[\omega J_{1}(\bar{k} a) G_{00}\right] .
$$

Similar to the 1D case, the two effective material parameters are given by

$$
\bar{\rho}=\bar{Z} \bar{k} / \omega, \quad \bar{C}=\omega \bar{Z} / \bar{k} .
$$

Also similar to the $1 \mathrm{D}$ case, the effective wave vector $\bar{k}$ is uniquely defined in Eq. (18a) by choosing the minimum $|\operatorname{Re}(\bar{k})|$. Evanescent waves arise if $G_{00} / G_{11}>0.054$ so that $\bar{k}$ would have imaginary parts. For the propagating waves $\left(G_{00} / G_{11} \leqslant 0.054\right)$, the direction of the phase velocity is also determined from Eq. (18a) by introducing a small dissipation into the system, following the similar arguments at the end of the previous section.

What one should be aware is that, owing to the truncation performed, the validity of Eq. (18) is conditioned on the monopole and dipole being the dominant modes. This is true only up to the frequency regime when the higher-order modes become significant. Within such a high-frequency range, although Eq. (18) can still accurately reproduce the monopolar and dipolar scattering behaviors, other components of the wave motions, such as those associated with the higher-order multipoles, can become much more dominant, and the validity of the scheme breaks down. However, the precise demarcation point is dependent on the precise microgeometry of the system and can vary from case to case.

It should be noted that, in maintaining the truncation at the dipole level, indirectly, we are also imposing some form of long-wavelength approximation, albeit in a weaker form. But such a weaker constraint has the advantage of giving the scheme more accuracy at somewhat higher frequencies.

\section{Results in the long-wavelength limit}

The homogenization results are much simpler if the condition of long effective wavelength, i.e., $\bar{k} a \ll 1$, is imposed. It would lead to results that are coincident with those obtained from the conventional method, such as the CPA [38,41].

By requiring $\bar{k} a \ll 1$, a set of explicit expressions can be obtained based on Eqs. (12) and (18),

$$
\begin{aligned}
\bar{k}_{\mathrm{L}} & = \pm \frac{1}{a} \sqrt{-d G_{11} / G_{00}}, \\
\bar{Z}_{\mathrm{L}} & = \pm \sqrt{-d G_{11} / G_{00}} /\left(\omega G_{11}\right), \\
\bar{\rho}_{\mathrm{L}} & =-d /\left(\omega^{2} a G_{00}\right), \\
\bar{C}_{\mathrm{L}} & =a / G_{11} .
\end{aligned}
$$

Here, the plus sign is chosen for conventional pass bands, the minus sign is chosen for negative refraction, and $d=1$ for $1 \mathrm{D}$ and $d=2$ for 2D. Notice that, in Eq. (19), the monopolar $G_{00}$ and dipolar $G_{11}$ each separately determine the effectivemass density $\bar{\rho}_{\mathrm{L}}$ and effective modulus $\bar{C}_{\mathrm{L}}$, respectively. This is noted to be not the case beyond the long-wavelength limit. Solutions of Eqs. (12) and (18) indicate that, in general, the
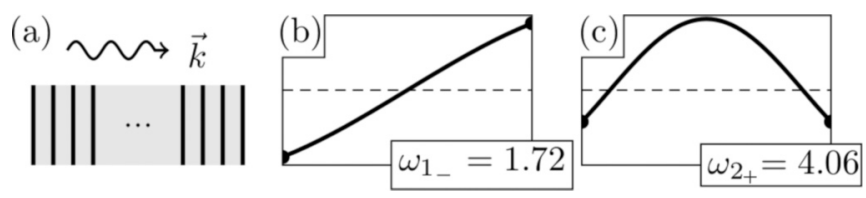

FIG. 2. (a) Schematic of the layered lattice. The thick black lines represent the stiff plates, whereas, the elastic layer is shaded in gray. Wave propagation is perpendicular to the plates. (b) and (c) Displacement fields for the first two nontrivial eigenstates of the relevant SU.

effective parameters $\bar{\rho}$ and $\bar{C}$ are each related to both $G_{00}$ and $G_{11}$.

\section{THREE EXAMPLES}

\section{A. One-dimensional layered lattice}

In this first example, we investigate a periodically laminated structure in which a stiff plate of negligible thickness alternates with an elastic layer, shown schematically in Fig. 2(a). This is a $1 \mathrm{D}$ system for waves propagating normal to the layers, and in this particular example, the displacement associated with the propagating waves is parallel to the layers, i.e., shear waves are considered. Since the system is $1 \mathrm{D}$, the procedure introduced in Sec. II B is applicable with the relevant $\rho$ and $C$ being the mass density and shear modulus, respectively.

We chose the SU containing one layer of the elastic material with two neighboring plates being its boundaries. The material parameters of the elastic material are set to be unity, i.e., $\rho_{0}=$ $1, C_{0}=1$, and the thickness is set to be 1 . By choosing the areal mass density of the stiff plate as $\rho_{A}=1$, the lowest two eigenmodes of the SU (excluding the first trivial mode of $\omega_{0_{+}}=0$, corresponding to rigid translation) are shown in Figs. 2(b) and 2(c). The first mode, at $\omega_{1_{-}}=1.72$, is dipolar in character; the second eigenmode, at $\omega_{2_{+}}=4.06$, is monopolar in character in which the two stiff plates vibrate in unison. The subscript + is used to denote the monopolar mode, and the dipolar mode is denoted by - .

Based on these eigenmodes, the relevant parameters $\rho_{i}$ [evaluated from Eq. (6)] $\omega_{i}$ and boundary displacements $\phi_{i}( \pm a)$ are shown in Table I. The two heterogeneous surface responses $G_{00}$ and $G_{11}$ can be evaluated from Eq. (9). We note that the rigid translation and the second mode at $\omega_{2_{+}}$ contribute only to $G_{00}$, whereas, the dipolar mode at $\omega_{1}$ contributes to $G_{11}$. An antiresonance can be found at $\tilde{\omega}_{1_{+}}=\pi$ at which $G_{00}=0$. Here, we use the tilde symbol to denote antiresonances. The four effective parameters $\bar{k}, \bar{Z}, \bar{\rho}$, and $\bar{C}$ in Eq. (12) can be calculated as functions of frequency with the results shown in Fig. 3 (ten eigenmodes have been

TABLE I. The values of $\omega_{i}, \rho_{i}$, and boundary displacement $\phi_{i}( \pm a)$ for each of the first three eigenstates in the layered lattice.

\begin{tabular}{lccrr}
\hline \hline$i$ & $\omega_{i}$ & $\rho_{i}$ & $\phi_{i}(a)$ & $\phi_{i}(-a)$ \\
\hline $0_{+}$ & 0 & 2.00 & 1.00 & 1.00 \\
$1_{-}$ & 1.72 & 3.70 & 1.64 & -1.64 \\
$2_{+}$ & 4.06 & 1.49 & -0.70 & -0.70 \\
\hline \hline
\end{tabular}




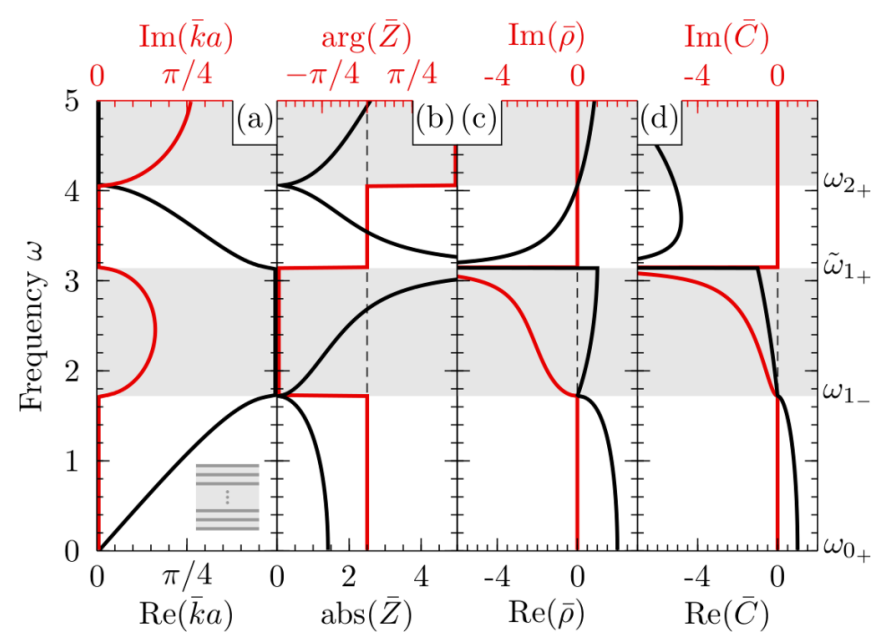

FIG. 3. (Color online) The effective parameters of the layered lattice. The black (red) curves represent the real (imaginary) parts of the effective parameters in (a), (c), and (d). In (b), the black curve denotes the amplitude, and the red curve denotes the phase. The band gaps are shaded in gray, whereas, the blank region denotes the pass bands. In the region $\left[\tilde{\omega}_{1_{+}}, \omega_{2_{+}}\right]$, the wave vector $\bar{k}$ is negative in value. However, it is plotted in the positive domain because of the usual convention.

employed here for comparison with simulations). Two band gaps (gray bands) appear in the frequency region $\left(\omega_{1_{-}}, \tilde{\omega}_{1_{+}}\right)$and at frequencies beyond $\omega_{2_{+}}$in which $\operatorname{Re}(\bar{C})<0$. A pass band with negative refraction of index can be found at $\left[\tilde{\omega}_{1_{+}}, \omega_{2_{+}}\right]$in which both $\bar{\rho}$ and $\bar{C}$ are negative in their real parts.

To check the validity of the effective description, we calculate the transmission through a length of this metamaterial embedded in a background medium. We set the background medium having material parameters $\rho_{\text {back }}=C_{\text {back }}=1$. From the effective parameters presented in Fig. 3, solutions of the transmission coefficient $T$ are analytically available from the transfer-matrix method [46]. The results for samples comprising different numbers of units are depicted in Fig. 4 as solid curves. They are seen to agree remarkably well with the direct simulation results (open circles). In Fig. 4(c), it is seen that the transmission intensity is dramatically reduced in the band gaps (the gray bands) when there are ten units. In contrast, when the sample comprises only a small number of units [Fig. 4(a)], the wave transmission and reflection are mainly determined by the impedance matching with the background medium [Fig. 3(b)].

As our homogenization scheme only matches the motion at the units' boundaries, the differences between the effective fields and the actual ones can be very pronounced inside the unit. To demonstrate this, transmission through a five-layer sample is calculated at three representative frequencies. The results are shown in Fig. 5. It is seen that, within the sample (the gray regions), the effective fields (black curves) only coincide with the actual motions (red curves) on the stiff plates (black points), i.e., the units' boundaries. The differences emerge inside the $\mathrm{SU}$ and can become significant at higher frequencies [see Fig. 5(c)]. Despite all these differences inside the SU, excellent agreement is seen between the homogenization prediction and the actual scattering fields in the background medium as expected.

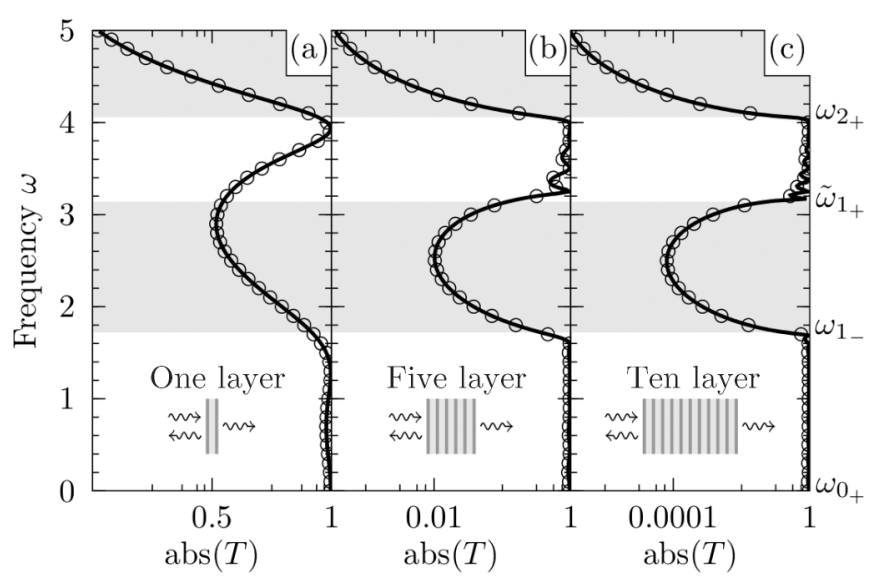

FIG. 4. Intensity transmission coefficients $T$ for the layered lattice samples comprising (a) one layer, (b) five layers, and (c) ten layers of the SU. The solid curves are the results predicted from the effective parameters in Fig. 3, and the open circles are the numerically simulated results. The band gaps are shaded in gray, whereas, the pass bands are shown as blank regions. Excellent agreement is seen between the homogenization predictions and the numerical simulations.

\section{B. Two-dimensional hexagonal lattice of concentric cylinders}

In the second example, we check the accuracy of our homogenization scheme for a $2 \mathrm{D}$ isotropic metamaterial. For simplicity, we consider a hexagonal lattice [Fig. 6(a)] with its high rotational symmetry [47]. The sample consists of cylindrical inclusions arranged in a triangular lattice in the $x-y$ plane, embedded in a uniform elastic matrix of $\rho_{0}=1$ and $C_{0}=1$. Each inclusion comprises three concentric cylinders. The elastic displacement of the wave is along the $z$ axis. By setting the lattice constant to be 1 , the radii of the three cylinders are $r_{1}=0.15, r_{2}=0.35$, and $r_{3}=0.4$, and the side length of the hexagonal unit cell is $l=1 / \sqrt{3}$.
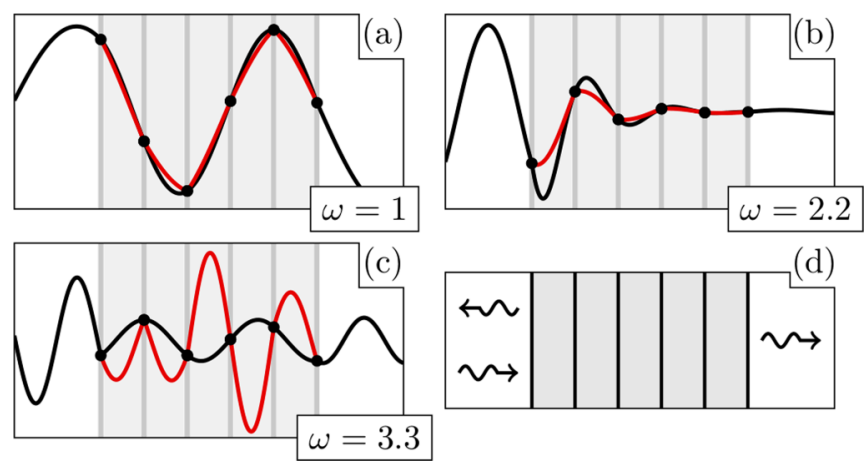

FIG. 5. (Color online) (a)-(c) The displacement fields for the layered lattice with five layers of the SU at three representative frequencies. The sample is shaded in gray. The black curves denote the effective fields obtained from homogenization prediction, whereas, the red lines represent the fields obtained by simulations. The black dots mark the displacements of the plates. It is seen that the homogenization theory cannot reproduce the internal displacement patterns of the metamaterial at higher frequencies, even though its prediction of the scattered field remains accurate. (d) Schematic of the incident, reflected, and transmitted waves for the system whose displacement field patterns are shown in (a)-(c). 

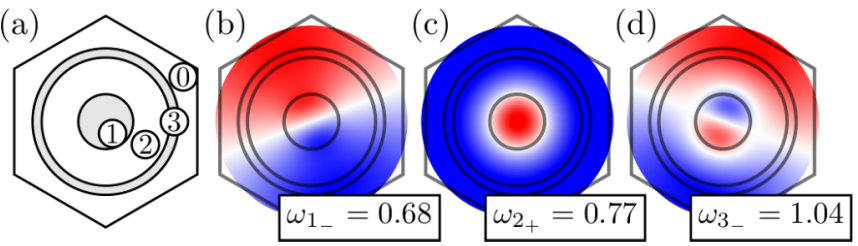

FIG. 6. (Color online) The unit cell and eigenstates of the hexagonal lattice. (a) The unit cell of the hexagonal lattice. Heavy components are colored gray, whereas, the light components are left blank. The numberings are for ease of identifying the different components within the unit cell. (b)-(d) The first three nontrivial eigenstates for the SU of the hexagonal lattice. Here, the SU is taken to be a circular region, whereas, the unit cell is hexagonal in shape. Such a difference is immaterial at low frequencies but can become noticeable at high frequencies, especially near the BZ boundaries.

For our purpose of homogenization, the $\mathrm{SU}$ is a cylinder with the filling ratio $v=0.58$, i.e., the same as that of the hexagonal unit cell [as in Figs. 6(b)-6(d)]. All components have same modulus $\left(C_{1}=C_{2}=C_{3}=C_{0}=1\right)$ but differ in their mass density: The central component is the heaviest with $\rho_{1}=400$, surrounded by a layer of material with $\rho_{2}=1$. The outermost layer of the inclusion has a mass density of $\rho_{3}=$ 100. By using the COMSOL MULTIPHYSICS FEM package, the lowest three nontrivial eigenstates of the SU are calculated as shown in Figs. 6(b)-6(d) (excluding the first rigid translation mode at $\omega_{0_{+}}=0$ ) with their frequencies at $\omega_{1_{-}}=0.68, \omega_{2_{+}}=$ 0.77 , and $\omega_{3_{-}}=1.04$.

Whereas, the symmetry of the second eigenmode is clearly monopolar and thereby only contributes to $G_{00}$, the first and third modes are dipolar in character and contribute to $G_{11}$. The surface responses can be calculated from Eq. (15) in which the values of the relevant parameters for each eigenmode are shown in Table II (the bra-ket notation here stands for the surface integral over the SU's surface). An antiresonance with $G_{00}=0$ can be found at $\tilde{\omega}_{1_{+}}=0.38$, whereas, another antiresonance is located at $\tilde{\omega}_{2_{-}}=0.82$ with $G_{11}=0$.

Solving Eq. (18) gives the values of the four effective parameters $\bar{k}, \bar{Z}, \bar{\rho}$, and $\bar{C}$ at each frequency as shown in Fig. 7 . We have used 25 eigenmodes for the relevant calculations. Three band gaps (gray bands) were found below $\omega=1.1$. In the first band gap $\left(\tilde{\omega}_{1_{+}}, \omega_{1_{-}}\right)$, the effective-mass density is negative in its real part; for the other two band gaps located at $\left(\omega_{2_{+}}, \tilde{\omega}_{2_{-}}\right)$and beyond $\omega_{3_{-}}$, the real part of effective modulus $\bar{C}$ is negative. A narrow negative index of the refraction pass band exists in the region $\left[\omega_{1_{-}}, \omega_{2_{+}}\right]$. The corresponding results from the CPA approach are also plotted with dashed lines for comparison. As seen in Fig. 7, the agreement is excellent at

TABLE II. The values of $\omega_{i}, \rho_{i}$, and boundary displacement $\left\langle\phi_{i} \mid \varphi_{0(1)}\right\rangle$ for each of the first four eigenstates in the hexagonal lattice.

\begin{tabular}{lcccc}
\hline \hline$i$ & $\omega_{i}$ & $\rho_{i}$ & $\left\langle\phi_{i} \mid \varphi_{0}\right\rangle$ & $\left\langle\phi_{i} \mid \varphi_{1}\right\rangle$ \\
\hline $0_{+}$ & 0 & 47.0 & 1.95 & 0 \\
$1_{-}$ & 0.68 & 18.4 & 0 & 2.41 \\
$2_{+}$ & 0.77 & 30.0 & 2.32 & 0 \\
$3_{-}$ & 1.04 & 12.7 & 0 & 0.45 \\
\hline \hline
\end{tabular}

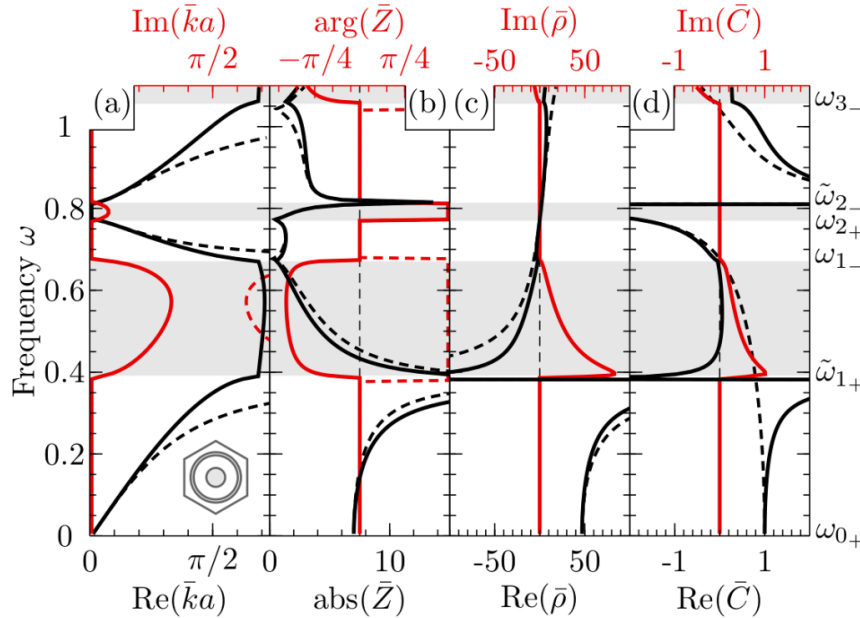

FIG. 7. (Color online) The effective parameters of the hexagonal lattice as deduced from the homogenization theory. The black (red) curves represent real (imaginary) parts of the effective parameters in (a), (c), and (d). In (b), the black curve denotes the amplitude, and the red curve denotes the phase of impedance. The band gaps are shaded in gray, whereas, the pass bands are left blank. For comparison, the results obtained from the CPA are plotted as dashed lines. It is seen that, at the limit of $\bar{k} a \ll 1$, the agreement with our scheme is very good. At higher frequencies, the present homogenization scheme is capable of reproducing the scattered fields rather accurately. It is seen that the CPA has failed to predict the imaginary parts in (c) and (d) as well as the resonant feature for the effective modulus at $\tilde{\omega}_{1_{+}}$.

the low-frequency regime, but the CPA differs significantly from our scheme when frequencies are away from the $\bar{k} a \ll 1$ limit. In particular, all the imaginary parts of the two effective material parameters $\bar{\rho}$ and $\bar{C}$ [Figs. 7(c) and 7(d)] as well as the resonant feature of $\bar{C}$ at $\tilde{\omega}_{1_{+}}[$Fig. $7(\mathrm{~d})]$ are missed by the CPA.

In order to verify our homogenization scheme, we compare the wave fields of a finite metamaterial sample with a similarly shaped homogeneous body having the relevant effective material parameters. As shown in Fig. 8(d), a sample consisting of 21 unit cells (the hexagons) is placed near a point source (red disk) in a medium with $\rho_{\text {back }}=C_{\text {back }}=1$. In Figs. 8(a)-8(c), we present the wave field comparisons under three representative frequencies. In each of these field mappings, in the lower half space is plotted the field arising from the heterogeneous metamaterial units, whereas, the predictions based on the homogenized units are plotted in the upper half. Both are calculated with the COMSOL MULTIPHYSICS FEM package. Clearly, the agreement is excellent in the external background medium as well as at the internal boundaries between adjacent unit cells (white lines). Our effective descriptions only fail in the internal regions of unit cells, especially at the central heavy cylinders. This comparison points out the essential difference between our scheme and the CPA. In the CPA, the intent is to agree everywhere; however, here, we focus only on the scattered field that is the basis of metamaterial's functionalities. We would like to note that, in Fig. 8(c), $\omega=1.022$ is very close to the Brillouin-zone boundary and is close to the third eigenmode frequency $\omega_{3_{-}}=1.04$, hence, resonant scattering features can be seen clearly. Here, the relevant wavelengths in the matrix and in the effective medium 


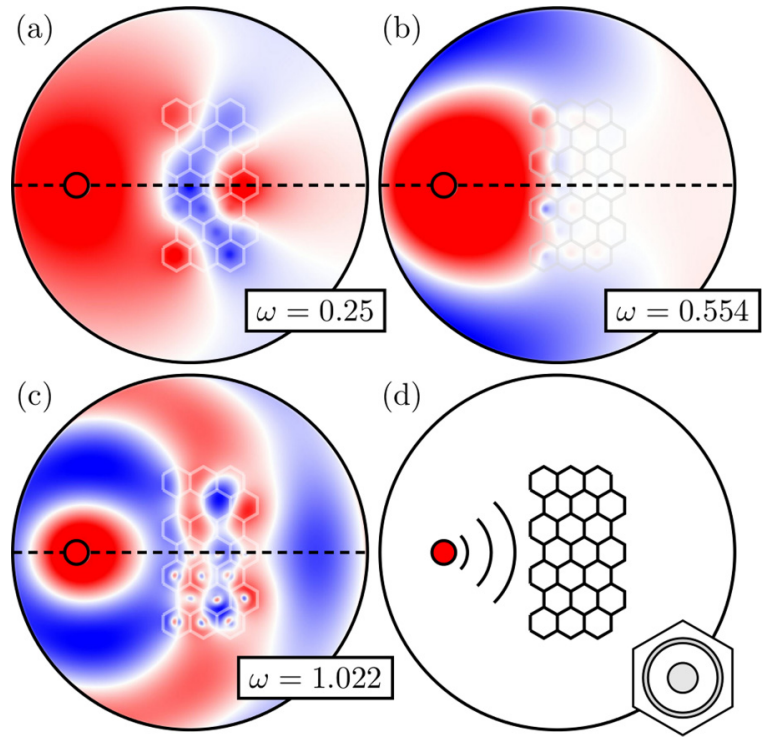

FIG. 8. (Color online) (a)-(c) The scattered fields for the hexagonal lattice with 21 units at three representative frequencies. The upper semicircular regions are the effective fields predicted from our homogenization scheme, and the lower semicircular regions are the results of numerically simulating the actual sample with all of its structural complexities. It is seen that, except for the inner regions of the metamaterial, the agreement is exceptionally good. (d) Schematic illustrating the relevant scattering process whose wave fields are shown in (a)-(c).

are only 6.15 and 1.96 times the lattice constant, respectively. Owing to the truncation performed, this effective description is expected to be inaccurate when the frequency reaches the first quadrupolar eigenmode frequency of the $\mathrm{SU} \omega=1.21$.

The anisotropy of the hexagonal lattice is expected to be important at the boundaries of the Brillouin zone. At these frequencies, the higher-order surface responses from the lattice's geometry are expected to play a role. This is clear in the band-structure comparison between the original heterogeneous hexagonal unit cell (open circles) and a homogenized one (solid lines) shown in Fig. 9. The former is based on the MST technique [48]. Besides the perfect agreement in most of the regions (except the "deaf" mode around $\omega=0.8$ that cannot be excited by propagating waves), discrepancies between the results of different approaches can be seen around the $M$ and $K$ points and can become significant at the $K$-point Dirac cone at $\omega=0.75$ (pointed by the arrow). This is a typical lattice scattering mode containing a quadrupole symmetric mode as shown in the right column of Fig. 9. In order to match such a lattice effect, anisotropic constitutive relations, containing more effective parameters, would have to be introduced. However, new effective parameters may bring new mechanisms into the original effective system. Although this can be interesting, further studies would be needed. These are left as a future pursuit.

\section{Decorated-membrane system}

The membrane-type metamaterial [21] is the third example for illustrating our scheme. The experimental sample comprises a rigid skeleton decorated by a planar array of designed

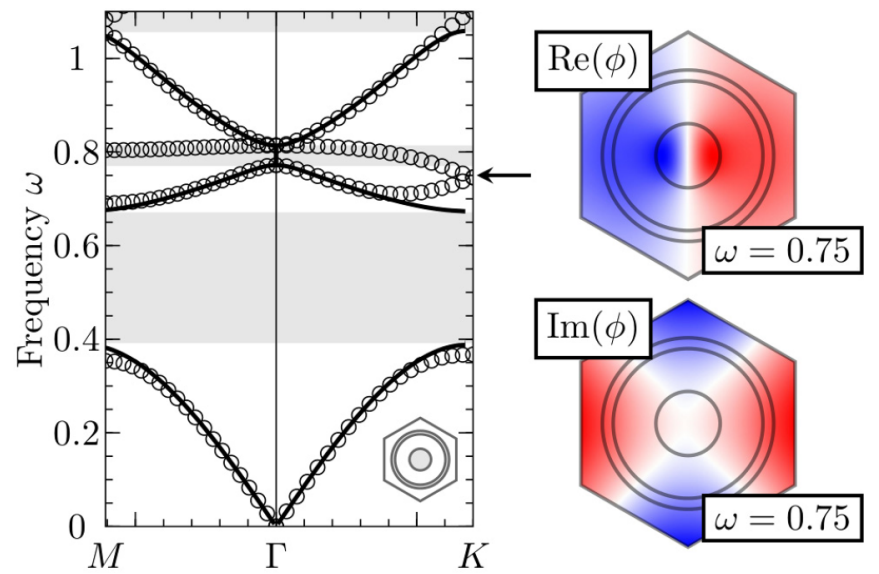

FIG. 9. (Color online) The band structures of the hexagonal lattice. The solid lines are predicted from our homogenization scheme, whereas, the open circles are the results of numerical simulations on the original heterogeneous composite. It is seen that the prediction of the homogenization scheme misses the band around $\omega=0.8$. This is the deaf mode that cannot be excited by propagating waves. The wave mode at the Dirac cone (marked by the arrow) is shown in the right panel.

membranes. In Fig. 10(a), we show a unit in the planar array, consisting of a circular elastic membrane (black line) with a radius of $R=14 \mathrm{~mm}$ and a thickness of $0.2 \mathrm{~mm}$, decorated by a circular rigid platelet with a radius of $4.6 \mathrm{~mm}$ and a mass of $237 \mathrm{mg}$ (delineated by the central flat rectangle) at the center. The membrane is fixed to an aluminum skeleton (indicated by two gray rectangles on the sides) with a radial tensile stress of $4.9 \times 10^{5} \mathrm{~Pa}$. The material parameters of the rubber membrane can be found in Ref. [49]. The unit cells are isolated from each other by the much higher rigidity of the aluminum grids as compared to the membranes. The amplitude and phase of the transmission and reflection were measured in a modified impedance tube apparatus, comprising two Brüel and Kjær-type-4206 impedance tubes with the sample sandwiched in between [50].

We limit the relevant acoustic angular frequency $\omega$ by the condition $2 \pi v_{0} / \omega=\lambda>2 R$, where $v_{0}=343 \mathrm{~m} / \mathrm{s}$ is the speed of sound in air. Thus, $\omega<7.70 \times 10^{4} \mathrm{~Hz}$ under this constraint. An immediate consequence is that, as far as the radiation modes are concerned, i.e., far-field transmission and reflection, the system can be accurately regarded as 1D and the conclusions in Sec. II B are applicable. This can be seen as the following. The normal displacement $W$ of the

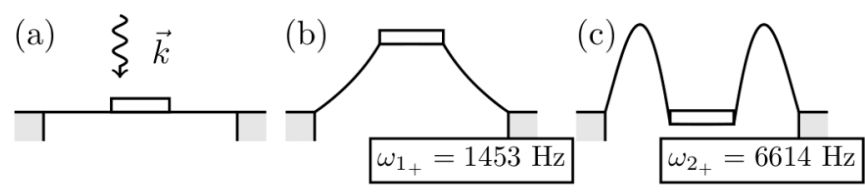

FIG. 10. (a) Schematic illustrating the decorated-membrane system. The black line represents the membrane, whereas, the central flat rectangle denotes the attached rigid platelet. The two gray rectangles on the sides denote the aluminum frame. (b) and (c) The first and second eigenmodes of the decorated membrane. Circular symmetry is assumed. 
membrane can be decomposed into $W=\langle W\rangle+\delta W$, where $\langle W\rangle$ represents the surface-averaged normal displacement of the membrane and $\delta W$ denotes the fine details of the membrane motion. In the air layer next to the membrane surface, the acoustic wave must satisfy the dispersion relation $k_{\|}^{2}+k_{\perp}^{2}=(2 \pi / \lambda)^{2}$, where $k_{\|(\perp)}$ represents the wave-vector component parallel (perpendicular) to the membrane surface. Because of the displacement continuity condition, the $2 \mathrm{D}\left\{k_{\|}\right\}$ set may be regarded as the Fourier-transform components of $W$. For the $\delta W$ component of the motion, we must have $k_{\|} \geqslant 2 \pi / 2 R>2 \pi / \lambda$, hence, the relevant $k_{\perp}^{2}<0$. That is, the displacement component $\delta W$ couples only to evanescent nonradiating modes. The displacement component $\langle W\rangle$, on the other hand, represents the pistonlike motion of the membrane and has $k_{\|}$components peaked at $k_{\|}=0$; hence, it is coupled to the radiation modes.

Figures 10(b) and 10(c) present the two eigenstates below the angular frequency of $7500 \mathrm{~Hz}$ that have the circular symmetry. For the first eigenmode at $\omega_{1_{+}}=1453 \mathrm{~Hz}$, the membrane and the rigid platelet vibrate in unison, whereas, in the second mode at $\omega_{2_{+}}=6614 \mathrm{~Hz}$, the membrane vibrates with the platelet remaining almost motionless. It is obvious that both eigenstates are monopolar and all the dipolar eigenstates are in the very high-frequency regime, owing to the small thickness of the membrane. One consequence is that the long-wavelength limit condition discussed in Sec. II D is now valid in almost the entire frequency range of interest (except for the very narrow regions around the antiresonances).

The effective bulk modulus $\bar{C}$ can be directly evaluated as $\langle C\rangle=2.68 \times 10^{9} \mathrm{~Pa}$. Based on the two eigenstates shown in Fig. 10, the other three effective parameters can be calculated from Eq. (19) in which

$$
\begin{aligned}
& G_{11}=a /\langle C\rangle, \\
& G_{00}=\sum_{i=1}^{2} \frac{2\left\langle W_{i}(a)\right\rangle^{2}}{\left\langle\rho_{i}\right\rangle\left(\omega_{i}^{2}-\omega^{2}-i \beta_{i} \omega\right)} .
\end{aligned}
$$

Here, $a=0.1 \mathrm{~mm}$ is the half thickness of the membrane, and $\left\langle\rho_{i}\right\rangle$ denotes the surface average of the $\rho_{i}$ in Eq. (6). Dissipative effects are considered in order to make comparisons with the experiments. Because the membrane's dissipative property is usually not available a priori, the values of the dissipation coefficients $\beta_{i}$ 's are extracted from the experimentally measured membrane displacement profile by using the method introduced in Ref. [29]. All the relevant parameters in Eq. (20) are available in Table III. The three effective parameters and their variations as functions of frequency are shown in Fig. 11. Five eigenstates were used in their evaluations.

It should be noted that the effective-mass density $\bar{\rho}$ diverges to negative infinite at the static limit [Fig. 11(c)]. This can

TABLE III. The values of $\omega_{i}, \rho_{i}$, surface-averaged boundary displacement $\left\langle W_{i}(a)\right\rangle$, and dissipation coefficient $\beta_{i}$ for each of the first two eigenstates in the decorated-membrane structure.

\begin{tabular}{lcccr}
\hline \hline$i$ & $\omega_{i}(\mathrm{~Hz})$ & $\left\langle\rho_{i}\right\rangle\left(\mathrm{kg} / \mathrm{m}^{3}\right)$ & $\left\langle W_{i}(a)\right\rangle$ & $\beta_{i}(\mathrm{~Hz})$ \\
\hline $1_{+}$ & 1453.1 & 7796 & 1.388 & 3.13 \\
$2_{+}$ & 6614.4 & 1379 & 1.424 & 30.02 \\
\hline \hline
\end{tabular}

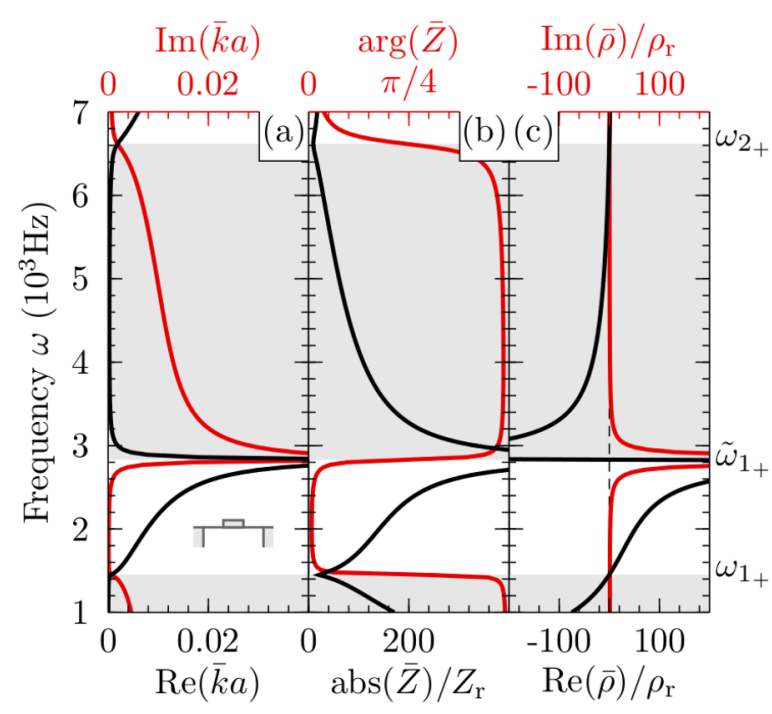

FIG. 11. (Color online) The effective parameters of the decorated-membrane structure. (a) The effective wave vector, (b) the effective impedance $\bar{Z}$, and (c) the effective mass density $\bar{\rho}$. The units for the material parameters are normalized to those of rubber: $Z_{\mathrm{r}}=1.9 \times 10^{5} \mathrm{~N} \mathrm{~s} \mathrm{~m}^{-3}$ and $\rho_{\mathrm{r}}=1.3 \times 10^{3} \mathrm{~kg} / \mathrm{m}^{3}$, respectively. The black (red) curves stand for real (imaginary) parts in (a) and (c). In (b), the black curve denotes the amplitude, whereas, the red curve denotes the phase. The band gaps are shaded in gray, whereas, the pass bands are left blank.

be understood as follows. At frequencies lower than the eigenfrequency, the structure response is always positive (in phase). As the rigid translational eigenmode at zero frequency makes no contribution to the membrane's response, the static limit behavior is dominated by the first eigenmode at $\omega_{1_{+}}$. The static surface-averaged displacement $\langle W(0)\rangle$ is thereby finite and in the same direction as the external forces. According to Newton's second law, the membrane would appear to have a divergent negative effective-mass density due to its infinitesimal but negative acceleration $\langle\ddot{W}(0)\rangle=-\omega^{2}\langle W(0)\rangle$ as $\omega \rightarrow 0$.

Within the frequency regime of interest, the two surface responses are usually different in their magnitudes, i.e., $G_{11} \ll$ $G_{00}$, except within the very narrow frequency range around the antiresonance $\tilde{\omega}_{1_{+}}=2830 \mathrm{~Hz}$. At the antiresonance, the monopolar $G_{00}$ approaches zero and becomes comparable with $G_{11}$. According to the discussion at the end of Sec. II B, a divergent imaginary effective wave vector can be expected when $G_{00}=G_{11}$ here [as shown in Fig. 11(a)]. This indicates an evanescent effective wave with infinitesimal decay distance in the membrane region. A very sharp dip in the transmission spectrum results as a consequence (Fig. 12). This is, indeed, observed.

The transmission and reflection for an airborne sound have been calculated (solid curves) from the effective parameters (shown in Fig. 11) based on the transfer-matrix method. The comparisons with the experimental measurements (open circles) are shown in Fig. 12 where the area of one unit cell is $50.3 \mathrm{~cm}^{2}$. Excellent agreement confirms the validity of our homogenization scheme. Notice a transmission dip can be found close to the antiresonance frequency $\tilde{\omega}_{1_{+}}$in which $\operatorname{Im}(\bar{k})=\infty$ if dissipation is ignored. 


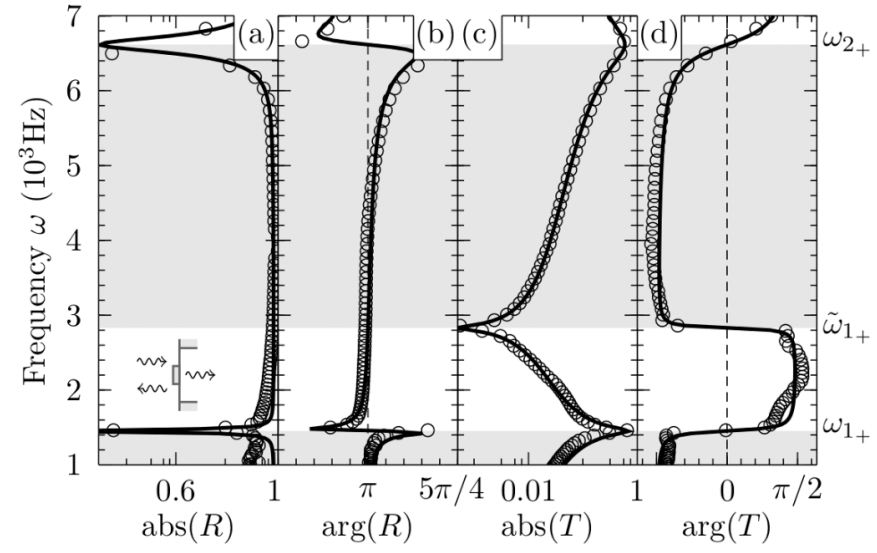

FIG. 12. (a) and (b) The reflection coefficient $R$ and (c) and (d) the transmission coefficient $T$ of the decorated-membrane structure for the airborne sound. The open circles are the experimental results obtained using the impedance tube measurements, whereas, the solid curves are calculated from the effective parameters shown in Fig. 11. The band gaps are shaded in gray, and the pass bands are without shading. Notice that the area of one unit cell is $50.3 \mathrm{~cm}^{2}$. Notice that the frequency here is the angular frequency $\omega=2 \pi \times f$. Excellent agreement is seen between the homogenization theory and the experimental results.

\section{CONCLUDING REMARKS}

We have developed a homogenization scheme for acoustic metamaterials based on reproducing the resonant scatterings of metamaterials rather than on minimizing scatterings. In our formalism, a finite volume of homogenized medium is described by the effective-mass density and modulus as functions of frequencies $\bar{\rho}=\bar{\rho}(\omega)$ and $\bar{C}=\bar{C}(\omega)$. This scheme provides an effective description for the macro/mesoscopic behaviors of the heterogeneous structures by exactly matching the motions at the boundaries between neighboring unit cells while ignoring the microscopic details inside. Employing the eigenmode's expansion for the SU, evaluation of its surface motions is facilitated by the resonance features of the metamaterials. This scheme demonstrates a broader validity compared to the traditional approaches. We have illustrated our scheme through its application to three examples. It is shown that the relevant effective description provided by this homogenization scheme is accurate for all frequencies for the 1D metamaterials and has excellent accuracy in the $2 \mathrm{D}$ case-easily extending to the regime near the Brillouin-zone boundaries. Application to the membrane-type metamaterials also has yielded excellent agreement.

Although our homogenization is based on matching the lowest two orders of resonant scatterings, applying it to the nonresonant metamaterials $[18,30]$ is equally straightforward, although the number of eigenmodes to be included might be large to attain the same accuracy.

Due to the similarity between the acoustic and the electromagnetic waves, one can easily apply the conclusions of this paper to EM problems through the simple translation,

$$
\text { displacement } \rightarrow E_{z}, \quad \rho \rightarrow \varepsilon, \quad \text { and } \quad C \rightarrow \mu^{-1},
$$

where $E_{z}$ is the polarized electric field perpendicular to the plane under considerations, $\varepsilon$ is the dielectric permittivity, and $\mu$ is the magnetic permeability.

\section{ACKNOWLEDGMENTS}

This work was supported by Hong Kong RGC Grants No. AoE/P-02/12, No. HKUST2/CRF/11G, and No. HKUST 606611.

\section{APPENDIX A: ONE-DIMENSIONAL SURFACE RESPONSES FOR A HOMOGENEOUS SAMPLE}

Consider a piece of homogeneous 1D material with length $2 a$, characterized by parameters $\bar{k}$ and $\bar{Z}$. The governing equations for the scalar wave $\bar{\phi}(x)$ are given by

$$
\left(\frac{\partial^{2}}{\partial x^{2}}+\bar{k}^{2}\right) \bar{\phi}(x)=0,
$$

accompanied by the boundary condition,

$$
\vec{\psi}(\xi)=\omega \bar{Z} \partial \bar{\phi}(x) /\left.\partial(\bar{k} x) \cdot \hat{n}\right|_{x=\xi},
$$

Here, $\hat{n}$ is the surface normal unit vector pointing outward, and $\bar{k}$ and $\bar{Z}$ are the relevant wave vector and impedance.

To calculate its monopolar surface response parameter $\bar{G}_{00}$, we introduce a set of monopolar surface tractions at its two ends,

$$
\vec{\psi}(a) \cdot \hat{n}=-\vec{\psi}(-a) \cdot \hat{n}=1 / \sqrt{2} .
$$

The consequent wave field inside is then

$$
\bar{\phi}(x)=-\frac{\cos (\bar{k} x)}{\sqrt{2} \omega \bar{Z} \sin (\bar{k} a)} .
$$

In accordance with its definition Eq. (3), the surface response $\bar{G}_{00}$ is given by

$$
\bar{G}_{00}=[\bar{\phi}(a)+\bar{\phi}(-a)] / \sqrt{2}=-\frac{\cot (\bar{k} a)}{\omega \bar{Z}} .
$$

Similarly, for the dipolar surface responses $\bar{G}_{11}$ with the surface tractions,

$$
\vec{\psi}(a) \cdot \hat{n}=\vec{\psi}(-a) \cdot \hat{n}=1 / \sqrt{2},
$$

the field is given by

$$
\bar{\phi}(x)=\frac{\sin (\bar{k} x)}{\sqrt{2} \omega \bar{Z} \cos (\bar{k} a)},
$$

and the surface response $\bar{G}_{11}$ is, therefore, $\bar{G}_{11}=$ $[\bar{\phi}(a)-\bar{\phi}(-a)] / \sqrt{2}=\tan (\bar{k} a) / \omega \bar{Z}$.

\section{APPENDIX B: TWO-DIMENSIONAL SURFACE RESPONSES FOR A HOMOGENEOUS SAMPLE}

The 2D scalar wave equation for $\bar{\phi}$ in an isotropic homogeneous material is given by

$$
\left(\frac{\partial^{2}}{\partial r^{2}}+\frac{1}{r} \frac{\partial}{\partial r}+\frac{1}{r^{2}} \frac{\partial^{2}}{\partial \theta^{2}}+\bar{k}^{2}\right) \bar{\phi}(r, \theta)=0,
$$

where $r$ and $\theta$ are the radial and angular coordinates, respectively, and $\bar{k}$ is the relevant wave vector. If the geometry 
of the domain under consideration is circular, the relevant surface normal vector $\hat{n}$ is always along the radial direction, and the boundary condition (16b) is given by

$$
\psi_{r}(a, \theta)=\omega \bar{Z} \partial \bar{\phi}(r, \theta) /\left.\partial(\bar{k} r)\right|_{r=a},
$$

with $a$ being the radius of the domain and $\bar{Z}$ being the relevant impedance.

The general solution $\bar{\phi}(r, \theta)$ for Eq. (B1) may be written as

$$
\bar{\phi}(r, \theta)=\sum_{n} A_{n} J_{n}(\bar{k} r) \cos (n \theta),
$$

with the relevant radial traction $\bar{\psi}_{r}(r, \theta)$ given by

$$
\begin{aligned}
\bar{\psi}_{r}(r, \theta) & =\omega \bar{Z} \partial \bar{\phi}(r, \theta) / \partial(\bar{k} r) \\
& =\frac{\omega \bar{Z}}{2} \sum_{n} A_{n}\left[J_{n-1}(\bar{k} r)-J_{n+1}(\bar{k} r)\right] \cos (n \theta) .
\end{aligned}
$$

Under a normal surface traction $\vec{\psi}(a, \theta)=\cos (\alpha \theta) \cdot \hat{n}$ on the circular boundary, the continuity of forces $\bar{\psi}_{r}(a, \theta)=\cos (\alpha \theta)$ at the boundary leads to the solution for coefficient $A_{n}$ in the general solution as

$$
A_{n}=2 \delta_{n \alpha} /\left\{\omega \bar{Z}\left[J_{\alpha-1}(\bar{k} a)-J_{\alpha+1}(\bar{k} a)\right]\right\} .
$$

Substitution of Eq. (B4) into Eq. (B3) gives the resultant wave fields inside as

$$
\bar{\phi}(r, \theta)=A_{\alpha} J_{\alpha}(\bar{k} r) \cos (\alpha \theta) .
$$

In accordance with its definition Eq. (3), the relevant nontrivial surface responses $\bar{G}_{\alpha \alpha}$ are then

$$
\begin{aligned}
\bar{G}_{\alpha \alpha} & =\frac{1}{\pi} \int_{0}^{2 \pi} \cos (\alpha \theta) \bar{\phi}(a, \theta) d \theta \\
& =A_{\alpha} J_{\alpha}(\bar{k} a) \\
& =\frac{2 J_{\alpha}(\bar{k} a)}{\omega \bar{Z}\left[J_{\alpha-1}(\bar{k} a)-J_{\alpha+1}(\bar{k} a)\right]} .
\end{aligned}
$$

Specifically, for $\bar{G}_{00}$ and $\bar{G}_{11}$ we have

$$
\begin{aligned}
\bar{G}_{00} & =-J_{0}(\bar{k} a) /\left[\omega \bar{Z} J_{1}(\bar{k} a)\right], \\
\bar{G}_{11} & =\frac{\bar{k} a J_{1}(\bar{k} a)}{\omega \bar{Z}\left[\bar{k} a J_{0}(\bar{k} a)-J_{1}(\bar{k} a)\right]} .
\end{aligned}
$$

[1] J. Li, L. Fok, X. Yin, G. Bartal, and X. Zhang, Nature Mater. 8, 931 (2009).

[2] A. Sukhovich, B. Merheb, K. Muralidharan, J. O. Vasseur, Y. Pennec, P. A. Deymier, and J. H. Page, Phys. Rev. Lett. 102, 154301 (2009).

[3] S. Zhang, L. Yin, and N. Fang, Phys. Rev. Lett. 102, 194301 (2009).

[4] J. Zhu, J. Christensen, J. Jung, L. Martin-Moreno, X. Yin, L. Fok, X. Zhang, and F. J. Garcia-Vidal, Nature Phys. 7, 52 (2010).

[5] C. M. Park, J. J. Park, S. H. Lee, Y. M. Seo, C. K. Kim, and S. H. Lee, Phys. Rev. Lett. 107, 194301 (2011).

[6] M. Farhat, S. Guenneau, and S. Enoch, Phys. Rev. Lett. 103, 024301 (2009).

[7] B. I. Popa, L. Zigoneanu, and S. A. Cummer, Phys. Rev. Lett. 106, 253901 (2011).

[8] S. Zhang, C. Xia, and N. Fang, Phys. Rev. Lett. 106, 024301 (2011).

[9] N. Stenger, M. Wilhelm, and M. Wegener, Phys. Rev. Lett. 108, 014301 (2012).

[10] H. Hu, A. Strybulevych, J. Page, S. E. Skipetrov, and B. A. van Tiggelen, Nat. Phys. 4, 945 (2008).

[11] N. Boechler, G. Theocharis, and C. Daraio, Nature Mater. 10, 665 (2011).

[12] B. Liang, X. Guo, J. Tu, D. Zhang, and J. Cheng, Nature Mater. 9, 989 (2010).

[13] B. Liang, B. Yuan, and J. C. Cheng, Phys. Rev. Lett. 103, 104301 (2009).

[14] J. Mei, G. Ma, M. Yang, Z. Yang, W. Wen, and P. Sheng, Nat. Commun. 3, 756 (2012).

[15] J. Christensen and F. J. G. de Abajo, Phys. Rev. Lett. 108, 124301 (2012).

[16] Y. Jing, J. Xu, and N. X. Fang, Phys. Lett. A 376, 2834 (2012).

[17] F. Liu, X. Huang, and C. T. Chan, Appl. Phys. Lett. 100, 071911 (2012).

[18] Z. Liang and J. Li, Phys. Rev. Lett. 108, 114301 (2012).
[19] R. Fleury and A. Alù, Phys. Rev. Lett. 111, 055501 (2013).

[20] Z. Liu, X. Zhang, Y. Mao, Y. Zhu, Z. Yang, C. T. Chan, and P. Sheng, Science 289, 1734 (2000).

[21] Z. Yang, J. Mei, M. Yang, N. H. Chan, and P. Sheng, Phys. Rev. Lett. 101, 204301 (2008).

[22] S. Lee, C. Park, Y. Seo, Z. Wang, and C. Kim, Phys. Lett. A 373 , 4464 (2009).

[23] S. Yao, X. Zhou, and G. Hu, New J. Phys. 12, 103025 (2010).

[24] N. Fang, D. Xi, J. Xu, M. Ambati, W. Srituravanich, C. Sun, and X. Zhang, Nature Mater. 5, 452 (2006).

[25] S. H. Lee, C. M. Park, Y. M. Seo, Z. G. Wang, and C. K. Kim, J. Phys.: Condens. Matter. 21, 175704 (2009).

[26] Y. Lai, Y. Wu, P. Sheng, and Z. Q. Zhang, Nature Mater. 10, 620 (2011).

[27] S. H. Lee, C. M. Park, Y. M. Seo, Z. G. Wang, and C. K. Kim, Phys. Rev. Lett. 104, 054301 (2010).

[28] Y. Wu, Y. Lai, and Z. Q. Zhang, Phys. Rev. Lett. 107, 105506 (2011).

[29] M. Yang, G. Ma, Z. Yang, and P. Sheng, Phys. Rev. Lett. 110, 134301 (2013).

[30] Y. Xie, B.-I. Popa, L. Zigoneanu, and S. A. Cummer, Phys. Rev. Lett. 110, 175501 (2013)

[31] G. Allaire and C. Conca, J. Math. Pures Appl. 77, 153 (1998).

[32] R. V. Craster, J. Kaplunov, and A. V. Pichugin, Proc. R. Soc. A 466, 2341 (2010).

[33] T. Antonakakis, R. V. Craster, S. Guenneau, and E. A. Skelton, Proc. R. Soc. A 470, 20130467 (2013).

[34] D. Smith and J. Pendry, J. Opt. Soc. Am. B 23, 391 (2006).

[35] M. G. Silveirinha, Phys. Rev. B 75, 115104 (2007).

[36] J. Willis, Mech. Mater. 41, 385 (2009).

[37] Y. Wu, J. Li, Z. Q. Zhang, and C. T. Chan, Phys. Rev. B 74, 085111 (2006).

[38] Y. Wu, Y. Lai, and Z. Q. Zhang, Phys. Rev. B 76, 205313 (2007)

[39] D. Torrent, A. Hakansson, F. Cervera, and J. Sanchez-Dehesa, Phys. Rev. Lett. 96, 204302 (2006). 
[40] D. Torrent and J. Saaanchez-Dehesa, Phys. Rev. B 74, 224305 (2006).

[41] D. Torrent and J. Sanchez-Dehesa, New J. Phys. 13, 093018 (2011).

[42] P. Sheng, in Introduction to Wave Scattering, Localization, and Mesoscopic Phenomena, 2nd ed., edited by R. Hull, R. M. Osgood, Jr., J. Parisi, and H. Warlimont, Springer Series in Materials Science Vol. 88 (Springer, Berlin, 2006).

[43] J. D. Jackson, Classical Electrodynamics, 3rd ed. (Wiley, New York, 1962), Chap. 1, p. 15.

[44] G. W. Milton and J. R. Willis, Proc. R. Soc. A 463, 855 (2007).
[45] J. R. Willis, in Continuum Micromechanics, edited by P. Suquet, CISM International Centre for Mechanical Sciences Vol. 377 (Springer, Berlin, 1997), p. 265.

[46] E. Adler, IEEE Trans. Ultrason. Ferroelectr. Freq. Control 37, 485 (1990).

[47] Y. Wu and Z. Q. Zhang, Phys. Rev. B 79, 195111 (2009).

[48] W. Zhang, C. T. Chan, and P. Sheng, Opt. Express 8, 203 (2001).

[49] T. Still, M. Oudich, G. K. Auerhammer, D. Vlassopoulos, B. Djafari-Rouhani, G. Fytas, and P. Sheng, Phys. Rev. B 88, 094102 (2013).

[50] K. Ho, Z. Yang, X. Zhang, and P. Sheng, Appl. Acoust. 66, 751 (2005). 